Annuaire suisse de politique de développement

26-2 | 2007

Financer le développement par la mobilisation des ressources locales

\title{
Comment conjurer la malédiction des ressources
} naturelles?

\section{Gilles Carbonnier}

\section{(2) OpenEdition}

\section{Journals}

Édition électronique

URL : http://journals.openedition.org/aspd/123

DOI : 10.4000/aspd. 123

ISSN : 1663-9669

Éditeur

Institut de hautes études internationales et du développement

Édition imprimée

Date de publication : 1 novembre 2007

Pagination : 83-98

ISBN : 978-2-88247-068-3

ISSN : 1660-5934

Référence électronique

Gilles Carbonnier, "Comment conjurer la malédiction des ressources naturelles ? », Annuaire suisse de politique de développement [En ligne], 26-2 | 2007, mis en ligne le 19 juin 2009, consulté le 10 décembre 2020. URL : http://journals.openedition.org/aspd/123 ; DOI : https://doi.org/10.4000/aspd. 123 


\title{
Comment conjurer la malédiction des ressources naturelles?
}

\author{
Gilles Carbonnier*
}

\section{Introduction}

L'Afrique enregistre sa plus forte croissance économique depuis vingt ans. Un rapport présenté à l'Assemblée annuelle de la Banque africaine de développement (BAD) à Shanghai le 20 mai 2007 estime que le taux de croissance moyen des économies africaines devrait atteindre $6 \%$ en $2007^{1}$. Cette accélération de la croissance s'explique notamment par une forte demande mondiale pour les matières premières - à commencer par le pétrole - et un investissement accru dans ces secteurs. Le rapport table sur une croissance exceptionnellement élevée pour les nouveaux pays producteurs de pétrole que sont la Mauritanie et le Soudan. Le taux de croissance de l'Angola pourrait même atteindre 27\% en 2007. Pour les économistes qui font rimer développement avec croissance économique, le constat positif est clair.

L'exploitation et le commerce des matières premières - notamment le pétrole représentent la principale source de devises étrangères et de recettes fiscales pour de nombreux pays en développement. Le secrétariat de l'Initiative pour la transparence des industries extractives (EITI) recense 53 pays « riches en ressources », soit des pays qui tirent plus du quart de leurs recettes budgétaires de l'activité extractive. Le Fonds monétaire international (FMI) indique dans une récente étude que les revenus pétroliers se montent à plus de $50 \%$ du revenu national brut (hors pétrole) des pays producteurs en 2005, contre 30\% en 2004 et $20 \%$ en $1999^{2}$. L'exploitation pétrolière fournit plus de $80 \%$ des recettes fiscales de pays comme l'Angola, la Guinée équatoriale ou le Nigeria ${ }^{3}$. Cette manne pourrait théoriquement leur permettre de financer tout ou partie de leurs efforts en matière de développement, ce d'autant que le prix du pétrole a été multiplié par sept depuis la fin des années 1990. Dans cette logique, il y aurait tout lieu de se réjouir de la découverte de nouveaux gisements dans des pays qui se trouvent en bas de l'échelle du développement humain. La demande accrue

* Professeur à l'Institut universitaire d'études du développement (IUED), Genève.

OECD, African Economic Outlook 2007, Paris, OECD, 2007.

2 IMF Fiscal Affairs Department, The Role of Fiscal Institutions in Managing the Oil Revenue Boom, Washington, DC, IMF, 5 March 2007.

3 Le pétrole fournit aussi une part substantielle des revenus à l'exportation. A titre d'exemple, trois quarts des recettes d'exportation du Soudan proviennent du pétrole. L'impact sur la balance courante n'est toutefois pas clair: l'exploitation pétrolière génère une hausse importante des importations de biens d'équipement et de consommation. Le FMI estime qu'une hausse des exportations pétrolières de $1 \%$ a été associée à une croissance de $0,8 \%$ des importations en Afrique subsaharienne sur la période 2003-2006, notamment en raison des investissements dans l'exploration et la production et les infrastructures pétrolières. Voir: FMI, Perspectives économiques régionales. Afrique subsaharienne, FMI, Washington, DC, avril 2007. 
de pétrole et de gaz génère en effet des flux financiers considérables pour un nombre croissant de pays à bas revenu, que ce soit en Afrique, en Asie ou en Amérique latine.

Or l'histoire nous enseigne que cet optimisme n'est pas de mise. La plupart des études récentes montrent que l'exploitation d'hydrocarbures tend à fragiliser le tissu économique, la cohésion sociale et les institutions politiques des pays producteurs. De manière paradoxale, l'exploitation des richesses du sous-sol est souvent associée à la misère des populations locales, à la mauvaise gouvernance et à la dégradation de l'environnement, ainsi que l'a exposé Terry Lynn Karl dans son ouvrage The Paradox of Plenty ${ }^{4}$. Quelques exceptions à la règle laissent pourtant espérer qu'il est possible de conjurer cette malédiction, pour autant qu'une série de conditions particulières soient réunies.

Dans cet article, nous nous intéressons d'abord à la malédiction des ressources naturelles et présentons quels traits saillants de l'évolution du marché pétrolier. Nous examinons ensuite les récentes initiatives qui ont pour objectif ultime de canaliser les revenus des activités extractives vers le financement du développement, notamment à travers une plus grande transparence et une meilleure gestion de la manne pétrolière.

\section{Le paradoxe de l'abondance}

Durant la guerre froide, le courant de pensée dominant considérait l'abondance de ressources naturelles comme un puissant vecteur de développement. Les investissements directs étrangers dans le secteur extractif et les recettes d'exportation devaient contribuer ipso facto au développement économique, conformément aux théories selon lesquelles l'apport de capital représentait la clé de voûte des stratégies de développement. Quelques économistes hétérodoxes y voyaient au contraire une source d'inégalités et de mal-développement, s'appuyant sur la thèse de Singer-Prebisch selon laquelle les termes de l'échange des matières premières tendent à se dégrader face aux produits manufacturés avec le temps. Or, l'orthodoxie a changé de camp à la fin des années 1980: une revue de la littérature récente sur la «malédiction des ressources naturelles» met en exergue l'impact négatif des ressources naturelles dans les pays en développement sur trois plans: $1^{\circ}$ la performance économique, $2^{\circ}$ le risque de guerre civile et $3^{\circ}$ le fonctionnement des institutions et la gouvernance 5 .

Les pays qui tirent une forte rente de l'exploitation de matières premières voient souvent la majorité de leur population sombrer dans la pauvreté et la précarité6. Le déclin espagnol au $\mathrm{XVI}^{\mathrm{e}}$ siècle est souvent cité comme exemple, alors que le pays exploitait les richesses du sous-sol latino-américain. Les

4 T.L. Karl, The Paradox of Plenty: Oil Booms and Petro-States, Berkeley, University of California Press, 1997, 342 p.

5 Pour une revue critique de la littérature, voir: A. Rosser, The Political Economy of the Resource Curse: A Literature Survey, IDS Working Papers, $n^{\circ} 268$, Brighton, Institute of Development Studies, 2006.

6 Voir par exemple: R. Auty (ed.), Resource Abundance and Economic Development, Oxford, Oxford University Press, 2001. 
causes de ce «paradoxe de l'abondance» sont multiples, comme le sont les explications relatives à l'impact négatif des ressources sur la performance économique des pays concernés. La hausse massive des recettes d'exportation de pétrole, par exemple, a pour effet l'appréciation de la monnaie nationale ${ }^{7}$. En conséquence, les autres secteurs d'exportation et d'activités soumis à la concurrence internationale perdent en compétitivité, avec des faillites et des pertes d'emploi à la clé. Cela tend à renforcer le mouvement de concentration économique autour du secteur extractif, qui est intensif en capital, mais très peu en main-d'œuvre: une fois les infrastructures en place, le fonctionnement des tours de forage et des plates-formes ne requiert que peu de personnel. C'est pourquoi un boom pétrolier ne permet pas d'absorber le chômage lié aux difficultés auxquelles doivent faire face d'autres secteurs économiques plus intensifs en main-d'œuvre (secteur textile par exemple). Il s'agit du «syndrome hollandais», vocable utilisé en référence aux difficultés économiques rencontrées par les Pays-Bas avec l'exploitation de gisements gaziers dans les années 1950 et $1960^{8}$.

Autre paradoxe, les gouvernements qui bénéficient de revenus accrus grâce à l'exploitation pétrolière ont tendance à creuser le déficit budgétaire. Les décideurs politiques engagent des dépenses publiques excessives, péchant par excès d'optimisme' , lorsqu'ils ne détournent pas purement et simplement la rente à leur profit dans une logique d'enrichissement personnel ou de clientélisme politique $^{10}$. Philip Lane et Aaron Tornell expliquent ces déficits budgétaires du fait que l'élite politique redistribue la rente à des groupes de pression influents de manière plus que proportionnelle à la croissance des revenus ${ }^{11}$. Les dépenses improductives augmentent, souvent dans le but de maintenir la paix sociale dans un contexte d'inégalités croissantes: un petit groupe profite de la manne pétrolière alors que la majorité n'en voit que les effets négatifs en termes de renchérissement du coût de la vie et de dégradation de l'environnement. Ce phénomène est aggravé dans les pays dont les institutions étatiques sont faibles et les groupes d'intérêt sectoriels puissants.

Le graphique ci-dessous illustre la corrélation entre hausse des recettes budgétaires tirées du pétrole et croissance des dépenses publiques dans le cas de quelques pays d'Afrique subsaharienne. Le Fonds monétaire international (FMI) estime que sur la période 2003-2006, une hausse de 1\% des recettes budgétaires tirées du pétrole a été associée à une croissance de $0,3 \%$ des dépenses budgétaires ${ }^{12}$.

7 A moins que la productivité de l'économie augmente de manière substantielle, ou qu'une partie de la rente pétrolière sorte du circuit économique.

8 Le syndrome hollandais ne concerne pas que les matières premières: un apport de ressources extérieures au titre de l'aide publique au développement ou de transferts de travailleurs migrants peut déployer les mêmes effets.

9 P. Mitra, Adjustment in Oil-Importing Developing Countries: A Comparative Economic Analysis, Cambridge, Cambridge University Press, 1994.

10 J.A. Robinson, R. Torvik and T. Verdier, Political Foundations of the Resource Curse, CEPR Discussion Paper Series, $n^{\circ}$ 3422, London, Center for Economic Policy Research (CEPR), June 2002.

11 P. Lane and A. Tornell, «Power, Growth, and the Voracity Effect», Journal of Economic Growth, vol. 1, nº 2, 1996, pp. 213-241.

12 FMI, Perspectives économiques régionales. Afrique subsaharienne, op. cit. 
Graphique: Pays exportateurs de pétrole d'Afrique subsaharienne - Dépenses budgétaires et recette pétrolières, 2002-2006 (variation annuelle en pourcentage)

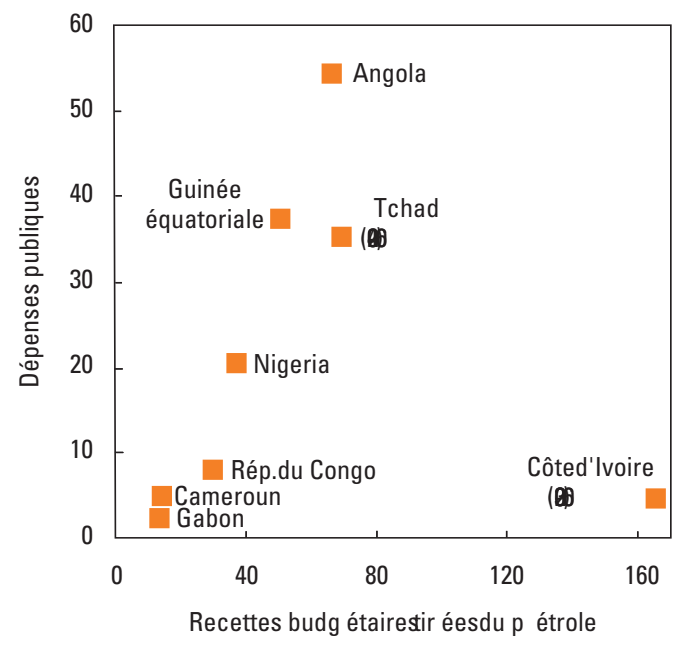

Source: FMI, Perspectives économiques régionales. Afrique subsaharienne, FMI, Washington, DC, avril 2007, p. 16

En ce qui concerne le risque de guerre civile, diverses études ont conclu que les tensions autour de la captation et la distribution de la rente augmentent l'instabilité politique et le risque de conflit armé. Paul Collier et Anke Hoeffler affirment sur la base d'analyses économétriques que les pays dont la prospérité repose sur l'exploitation de matières premières présentent un risque accru de guerre civile, notamment lorsque ces activités génèrent au moins un tiers du revenu national brut $^{13}$. Une étude réalisée par le Royal Institute of Foreign Affairs (Chatham House) en 2005 ajoute que les pays dont la production quotidienne de pétrole par habitant se situe entre 5,5 et 24 barils tendent à souffrir d'une faible capacité de gouvernance, d'instabilité politique et de conflits armés ${ }^{14}$. Au-dessus de 24 barils par jour et par habitant, la production de pétrole aurait un effet bénéfique global pour la population (exemple du Koweit) par le biais d'une réduction de la pauvreté monétaire pour la grande majorité de la population. Audessous de 5,5 barils, les revenus seraient trop faibles pour avoir un impact significatif (exemple de la Mauritanie). L'on trouve aujourd'hui dans la «zone dangereuse » des pays tels que l'Angola, la République du Congo et le Nigeria. D'autres pays s'en approchent en raison d'une production en hausse (exemple du Soudan) ou en baisse (le Gabon).

Quant aux aspects politiques et institutionnels, des auteurs tels que Nathan Jansen et Leonard Wantchekon estiment qu'il existe une corrélation entre l'abondance de ressources naturelles et les régimes autocratiques en Afrique ${ }^{15}$. Ils

13 P. Collier and A. Hoeffler, «On Economic Causes of Civil War», Oxford Economic Papers, ${ }^{\circ}$ 50, 1998, pp. 563-573.

14 K. Myers, Petroleum, Poverty and Security, Chatham House Briefing Papers, London, Royal Institute of International Affairs, June 2005.

15 N. Jensen and L. Wantchekon, «Resource Wealth and Political Regimes in Africa», Comparative Political Studies, n 377, 2004, pp. 816-841. 
rejoignent Terry Lynn Karl pour montrer que la manne pétrolière permet aux gouvernements de réaliser des projets d'investissement pharaoniques ou d'engager des dépenses en faveur d'un groupe d'intérêt sans devoir se soucier de soigner leurs relations avec les citoyens-contribuables ni de leur rendre des comptes.

Les activités de l'industrie extractive et la consommation d'hydrocarbures ont un impact déterminant sur l'environnement. Les installations de pompage, les pipelines, les raffineries et les infrastructures de commercialisation se traduisent trop souvent par des déforestations, des déplacements forcés de populations, des pollutions et d'autres atteintes graves à l'environnement. La consommation de pétrole est l'un des facteurs majeurs du réchauffement planétaire. Dans cet article, nous n'aborderons pas ces questions essentielles liées à l'environnement mais référons le lecteur à l'article de Wolfgang Sachs paru dans le dossier 2006 de l'Annuaire suisse de politique de développement sous le titre «De l'huile sur le feu. La lutte pour les ressources attise l'insatisfaction planétaire ${ }^{16}$.

En résumé, une majorité d'études récentes incitent au pessimisme ${ }^{17}$. La plupart des études de cas accréditent aujourd'hui la thèse de la malédiction des matières premières en général, et du pétrole en particulier. Cette conclusion n'est guère réjouissante pour les pays à faible revenu qui commencent à exploiter de nouveaux gisements afin de répondre à une demande mondiale en forte croissance, comme nous le verrons ci-après. Il existe toutefois des exceptions à la règle. Quelques pays en développement ont su tirer parti de la richesse de leur soussol pour lutter contre la pauvreté avec un succès relatif (par exemple le Botswana, l'Indonésie et la Malaisie). Pour Paul Stevens, l'explication se trouve dans les politiques économiques adoptées par les autorités: prudence fiscale et budgétaire, efforts de diversification économique et soutien au secteur agricole, politique de taux de change visant à empêcher l'appréciation de la monnaie, bureaucratie compétente, etc. ${ }^{18}$ Les liens de causalité entre ressources naturelles et développement sont complexes et l'on ne saurait tirer de conclusion définitive et universelle.

\section{Des hydrocarbures toujours plus convoités}

La croissance annuelle moyenne de la consommation mondiale d'énergie avait tendance à osciller autour de 1,4\% depuis les années 1970. Le boom économique chinois et indien a renforcé cette croissance qui a atteint 2,8\% en 2004 et $4 \%$ en $2005^{19}$. Résultat: le prix des matières premières s'envole sur les marchés

16 W. Sachs, «De l'huile sur le feu. La lutte pour les ressources attise l'insatisfaction planétaire», in C. Schümperli Younossian (dir.) et X. Tschumi (resp. de dossier), Paix et sécurité. Les défis lancés à la coopération internationale, Annuaire suisse de politique de développement, vol. 25, $\mathrm{n}^{\circ} 2$, Genève, IUED, 2006, pp. 129-138.

17 Voir aussi : C. Leite and J. Weidmann, Does Mother Nature Corrupt?, IMF Working Papers, $\mathrm{n}^{\circ}$ 99/85, Washington, DC, 1999. M. Ross, «The Political Economy of the Resource Curse», World Politics, vol. 51, nº 2, 1999, pp. 297-322.

18 P. Stevens, «Resource Impact: A Curse of a Blessing ?», CEPMLP Internet Journal, vol. 14, article 1, Dundee, Dundee University, 2003, <http://www.dundee.ac.uk/cepmlp/journal/html/Vol14/article14_1. html $>$ (consultation: 7 juin 2007).

19 P. Chalmin (dir.), Cyclope 2006. Les marchés mondiaux, Paris, Economica, 2006. 
mondiaux. Le prix du baril de pétrole, qui était passé sous la barre des 10 dollars en 1999, a dépassé les 70 dollars à l'été 2006. Ce mouvement haussier devrait encore se renforcer. L'Agence internationale de l'énergie (AIE) table sur une hausse de la demande mondiale d'énergie de plus de $50 \%$ d'ici à 2030. La consommation de pétrole pourrait augmenter de $40 \%$ environ, ce qui ne manquera pas d'accentuer la pression sur les prix dans la prochaine décennie. Pour l'AIE, la dépendance des pays de l'Organisation de coopération et de développement économiques (OCDE) à l'égard des pays producteurs du Moyen-Orient ira en s'accentuant: "La concentration de la production pétrolière dans un petit nombre de pays dotés de réserves considérables - notamment les pays du Moyen-Orient membres de l'OPEP et la Russie - confortera leur position dominante sur le marché et leur latitude d'imposer des prix plus élevés. » ${ }^{20}$

Pour contrer ce risque, les grands pays importateurs cherchent à diversifier leurs sources d'approvisionnement. Les sociétés pétrolières chinoises, indiennes, malaisiennes, européennes et nord-américaines investissent des milliards de dollars dans l'exploration pétrolière, la construction d'oléoducs et l'exploitation de nouveaux gisements dans un nombre croissant de pays en développement (Colombie, Côte d'Ivoire, Equateur, Guinée-Bissau, Guinée équatoriale, Mauritanie, Myanmar, Papouasie-Nouvelle-Guinée, Soudan, Tchad, Timor-Leste, etc.). Une première en 2006, l'Afrique a dépassé le Moyen-Orient en tant que fournisseur de pétrole aux Etats-Unis, dont $26 \%$ des importations ont été fournies par l'Algérie, l'Angola et le Nigeria contre $23 \%$ par l'Arabie saoudite, l'Irak et le Koweït. Toutefois, les réserves prouvées de pétrole sont nettement plus abondantes au Moyen-Orient que sur le continent africain, où quelques pays producteurs auraient déjà atteint leur «pic de production», à l'instar du Gabon en 1997.

La manne pétrolière permettra-t-elle à ces nouveaux pays producteurs d'atteindre plus facilement les Objectifs du Millénaire pour le développement? Dans quelle mesure les investissements dans le secteur des hydrocarbures se traduiront-ils par des moyens financiers accrus en faveur du développement économique et social? Plusieurs initiatives ont récemment vu le jour dans le but de briser le cercle vicieux entre matières premières et mal-développement. Ces initiatives réunissent dans d'improbables alliances des acteurs aux cultures et aux objectifs divergents: Banque mondiale et Fonds monétaire international, organisations non gouvernementales (ONG) de défense des droits humains, investisseurs privés, agences gouvernementales de coopération au développement, gouvernements de pays producteurs, etc. Elles bénéficient du soutien politique de quelques pays industrialisés producteurs de pétrole (Norvège, Royaume-Uni) qui ont su conjurer la malédiction de l'or noir. Ces pays ambitionnent de partager leur expertise dans ce domaine, même s'il va de soi que leurs caractéristiques sociopolitiques, démographiques, culturelles et institutionnelles diffèrent fondamentalement de celles rencontrées dans les nouveaux eldorados pétroliers d'Afrique subsaharienne.

20 International Energy Agency (IEA), World Energy Outlook 2006. Résumé et conclusions, Paris, IEA, 2006, p. 3 . 
La littérature sur la malédiction des ressources naturelles ainsi que sur l'évolution des marchés des matières premières ne pouvait laisser la communauté internationale indifférente. Dès la fin des années 1990, certaines ONG ont dénoncé le pillage de ressources naturelles par des régimes prédateurs avec la complicité de l'industrie extractive et du secteur financier. Le Conseil de sécurité des Nations unies a mandaté plusieurs groupes d'experts pour faire rapport sur l'exploitation illégale de ressources naturelles ainsi que sur la violation d'embargos décrétés à l'encontre de pays déchirés par la guerre civile.

La question du financement des guerres civiles a fait l'objet d'une attention particulière depuis le milieu des années 1990. Sous l'impulsion de diverses ONG (Partnership Africa-Canada, Global Witness), les pays importateurs et exportateurs de diamants ont établi un système de certification dans le cadre du Processus de Kimberley. Il s'agit d'une initiative novatrice dans laquelle l'industrie du diamant, plus de 70 gouvernements ainsi que des ONG ont établi un système visant à garantir que les diamants ne soient pas utilisés par des mouvements rebelles pour financer la guerre contre des gouvernements légitimes. Le système fonctionne depuis janvier 2003 et interdit tout commerce de diamants bruts avec des pays qui ne participent pas à l'initiative. Tous les chargements de diamants bruts doivent être accompagnés de certificats d'origine. Les pays qui ne se conforment pas aux obligations et aux modalités de certification peuvent se voir exclus du processus, c'est-à-dire du marché (légal) du diamant.

L'obligation de fournir un certificat pour toute exportation de diamants bruts a permis par ailleurs d'augmenter la valeur des exportations officielles dans les pays où une part importante du commerce passait par les circuits informels ou illégaux. Plusieurs pays producteurs ont ainsi vu les retombées fiscales de l'exploitation de diamants augmenter. Toutefois, le système de Kimberley n'est pas un instrument de développement. Il ne permet pas d'améliorer le sort de centaines de milliers de creuseurs de diamants sur le terrain. C'est pourquoi des ONG membres du Processus de Kimberley ont lancé la Diamond Development Initiative à Accra en octobre 2005 avec le soutien de l'industrie et de gouvernements, afin d'améliorer les conditions de travail et la rémunération des mineurs à travers la formation, l'accès au crédit et la fourniture d'équipements notamment.

Les pays membres du Processus de Kimberley ont obtenu une dérogation aux règles de l'Organisation mondiale du commerce (OMC) en février 2003 par une décision du Conseil général de l'organisation. Cette dérogation leur permet de se soustraire au principe de la nation la plus favorisée pour fixer des restrictions quantitatives à l'importation, et ce de manière discriminatoire à l'encontre de pays soupçonnés d'alimenter le commerce des «diamants de la guerre». Le Conseil du commerce des marchandises de l'OMC a justifié cette dérogation aux principes fondamentaux du système commercial multilatéral en invoquant «le caractère humanitaire exceptionnel de cette question, les effets dévastateurs des conflits alimentés par le trafic des diamants de la guerre sur la paix et la sécurité des populations des pays touchés, ainsi que les violations graves et systématiques des droits de l'homme qui sont commises 
lors de tels conflits ${ }^{21}$. Fin 2006 , cette dérogation a été reconduite pour une nouvelle période de six ans ${ }^{22}$.

La Banque mondiale a lancé en juillet 2001 une réflexion sur l'opportunité de poursuivre ses programmes de prêts pour des projets d'extraction, la Revue des industrie extractives (Extractive Industries Review). Cette revue, menée par un panel d'experts sous la présidence d'Emil Salim, ex-ministre indonésien de l'Environnement, a permis d'engager un dialogue entre la Banque mondiale et d'autres parties prenantes concernées par la problématique: gouvernements, organisations de peuples indigènes, syndicats, industrie, milieux académiques, etc. La revue a conclu en novembre 2003 que la Banque mondiale pouvait encore jouer un rôle dans le secteur extractif, mais seulement dans la mesure où son intervention permettait d'assurer que les projets d'extraction contribuent à lutter contre la pauvreté et à promouvoir un développement durable. Nous verrons ci-après que l'exemple du Tchad remet en cause la capacité de la Banque mondiale à s'attaquer à la malédiction des ressources.

Certaines agences gouvernementales de coopération au développement ont fait de la bonne gestion de la manne pétrolière un axe d'action important. Il n'est pas surprenant que deux pays exportateurs d'hydrocarbures aient pris l'initiative, forts de leur expérience en matière de gestion de la rente pétrolière. Le Département britannique du développement international (DFID) a joué un rôle central dans la conception et la mise en œuvre de l'Initiative pour la transparence des industries extractives (voir ci-après). L'Agence norvégienne pour la coopération en faveur du développement (NORAD) a lancé un programme pour aider les pays producteurs du Sud à transformer les revenus pétroliers en meilleures conditions de vie pour la population (Oil for Development). Le NORAD fournit ainsi des conseils en matière de gestion des ressources et des revenus ainsi que de protection de l'environnement à des pays comme l'Angola, le Cambodge, l'Irak, le Soudan et le Vietnam.

Le secteur privé a lui aussi réagi. De nombreuses sociétés multinationales ont adopté des codes de conduite et des lignes directrices relatifs à l'environnement, aux droits humains, aux conditions de travail et aux relations avec les communautés locales. Les débats sur la responsabilité sociale de l'entreprise ne portent pas seulement sur la nature volontaire ou juridiquement contraignante des engagements pris par les sociétés privées, mais aussi sur des questions éthiques complexes. Il s'agit notamment de mieux cerner les contours et les limites de la responsabilité de l'industrie extractive dans des domaines tels que la fixation des prix et les arrangements de partage des revenus avec les Etats hôtes, la création d'emplois non précaires, le rapatriement des profits ou encore l'évasion fiscale via des sociétés offshore.

21 OMC, Accord sur une dérogation pour les «diamants de la guerre» dans le cadre de l'OMC, 26 février 2003, <http://www.wto.org/french/news_f/news03_f/goods_council_26fev03_f.htm> (consultation: 7 juin 2007).

22 OMC, Le Conseil du commerce des marchandises examine un nouvel élargissement des CE et proroge une dérogation concernant le système de Kimberley, 20 novembre 2006, <http://www.wto.org/ french/news_f/news06_f/ctg_20nov06_f.htm> (consultation: 7 juin 2007). 
Les conditions contractuelles négociées entre Etats et entreprises pétrolières déterminent le volume des recettes budgétaires pour les pays producteurs. La hausse du prix du pétrole a incité plusieurs gouvernements ${ }^{23}$ à exiger un meilleur partage des profits par le biais d'une révision des contrats et des arrangements fiscaux conclus avec les firmes pétrolières.

Sur cette toile de fond, la transparence des revenus est censée permettre aux citoyennes et citoyens d'exiger des comptes quant à l'usage que leur gouvernement fait de la manne pétrolière, réduisant ainsi les risques de détournement de fonds et de corruption. Une allocation budgétaire judicieuse doit ensuite permettre de réduire les principaux risques associés à l'exploitation des ressources, qu'ils soient de nature économique (syndrome hollandais, manque de diversification) ou politique (répartition inéquitable des revenus contribuant à l'instabilité, voire au conflit armé). Dans la pratique, il est impossible pour des acteurs de la société civile d'appréhender l'ensemble des paiements et des revenus perçus par un gouvernement au titre de la production et de la vente d'hydrocarbures. Les contrats de partage de production et les systèmes de redevances sont opaques et complexes. Ces questions font maintenant l'objet de modules de formation, de mandats de consultants et de publications à l'attention du public ${ }^{24}$.

\section{$\square$ Publiez ce que vous payez}

L'ONG britannique Global Witness a été la première à mener des campagnes publiques pour améliorer la transparence relative à l'exploitation des ressources naturelles et la gestion des revenus y afférents. Dans son rapport A Crude Awakening, publié en décembre 1999 à Luanda, Global Witness dénonçait la complicité du secteur pétrolier et financier dans le système de captation de la rente par la présidence angolaise au détriment de la population. Ce souci rejoignait les préoccupations du FMI, qui mettait comme condition à la reprise de ses programmes un audit externe des comptes de l'Etat angolais.

Avec d'autres $\mathrm{ONG}^{25}$, Global Witness a contribué au lancement de la campagne «Publiez ce que vous payez» (PCQVP) en juin 2002, à l'initiative du financier George Soros, président de l'Open Society Institute. PCQVP exige que toutes les compagnies multinationales et les entreprises d'Etat publient à titre individuel les chiffres annuels nets des paiements qu'elles versent aux gouvernements. Ces revenus incluent notamment les redevances perçues sur la valeur de la production, les bonus versés à la signature des contrats d'exploitation, l'impôt sur les bénéfices des sociétés après déduction des coûts et amortissements liés aux investissements nécessaires à l'exploration et l'exploitation, l'impôt à la source sur les paiements de dividendes, les droits de douane, la taxe sur la valeur ajoutée, etc. PCQVP demande que ces informations soient mises à la disposition de la société civile, en langues et monnaie locales. La coalition exige

23 Par exemple l'Algérie, la Bolivie, la Russie et le Venezuela.

24 Par exemple J. Shultz, Suivez l'argent! Guide de surveillance des budgets publics et des revenus du pétrole et du gaz, New York, Open Society Institute, 2005.

25 CAFOD, Oxfam UK, SCF UK, Transparency International UK, CRS, Human Rights Watch, Pax Christi NL, Caritas France, etc. 
que les chiffres soient vérifiés par des organismes indépendants multi-parties prenantes (multi-stakeholders) ou par le FMI.

La campagne PCQVP ne vise pas directement les gouvernements des pays producteurs. Elle exerce plutôt une pression sur des acteurs influents capables d'inciter ces gouvernements à introduire plus de transparence dans ce domaine sensible. La campagne vise en particulier la Banque mondiale, le FMI, les autorités boursières et les banques commerciales afin qu'ils fassent pression tant sur l'industrie extractive que sur les pays producteurs pour que l'obligation de transparence des revenus prime sur les clauses de confidentialité26.

\section{$\square$ L'Initiative pour la transparence de l'industrie extractive}

La campagne PCQVP a réussi à attirer l'attention des décideurs politiques et du public sur la problématique de la transparence liée à la rente minière et pétrolière. Tony Blair a annoncé le lancement d'une initiative multi-parties prenantes sur ces questions lors du Sommet mondial sur le développement durable à Johannesburg en octobre 2002. L'année suivante, les pays du G-8 appelaient les gouvernements et les entreprises à rendre public le montant de leurs paiements, dans une déclaration commune lors du sommet d'Evian. Le 17 juin 2003, le gouvernement britannique lançait l'Initiative pour la transparence des industries extractives (en anglais: Extractive Industries Transparency Initiative, EITI) lors d'une conférence réunissant l'industrie extractive, les organisations non gouvernementales, les gouvernements de pays consommateurs et producteurs, des organisations internationales ainsi que le secteur financier. Les participants ont adopté des principes communs sur la transparence des revenus générés par les activités d'extraction. Le DFID a joué un rôle central dans la mise en œuvre de ce processus et en a assuré le secrétariat.

Les parties prenantes se sont accordées dès 2003 sur l'objectif de renforcer la bonne gouvernance dans des pays riches en ressources naturelles grâce à la publication et à la vérification des paiements effectués par les entreprises et des revenus perçus par les gouvernements sur les activités extractives. Il s'agit de développer des mécanismes de transparence dans tous les pays en développement riches en ressources naturelles, c'est-à-dire dont les recettes budgétaires proviennent pour plus d'un quart de l'activité extractive. Les modalités de mise en œuvre de l'EITI au niveau national n'ont été précisées qu'en mars 2005: les entreprises qui participent à l'initiative et exploitent des ressources dans un pays signataire doivent déclarer tous les paiements versés au gouvernement, au moins sous forme globale ou consolidée. De son côté, le gouvernement doit publier régulièrement les paiements reçus des sociétés privées et des entreprises d'Etat pour permettre une comparaison ou «un rapprochement» des chiffres fournis par les compagnies avec ceux déclarés par le gouvernement. Ce dernier doit aussi publier les autres revenus issus de l'exploitation et du commerce des produits d'extraction. Tous ces chiffres doivent être audités par un «système crédible et indépendant». La société civile doit pouvoir participer à la conception, au contrôle et à l'évaluation du processus ainsi qu'au débat public sur les revenus du secteur extractif. Il est proposé de nommer dans chaque pays producteur un «administrateur crédible et indépendant» dont le rôle est de renforcer la crédibilité du processus sur le plan national.

26 Voir le site Internet de PCQVP: <http://www.publishwhatyoupay.org>. 
Quels sont les résultats? Un rapport publié par PCQVP et le Revenue Watch Institute en octobre 2006 sous le titre Un regard sur l'EITI ${ }^{27}$ reconnaît certes quelques succès: 21 gouvernements de pays africains, asiatiques et latino-américains ont rejoint l'EITI entre 2003 et 2006 (sur un total de 53 pays riches en ressources). Mais seuls deux d'entre eux ont publié des rapports audités, à savoir le Nigeria et l'Azerbaïdjan. Huit pays n'avaient pas encore nommé de responsable à la tête du processus EITI, dix n'avaient pas formé de comité multi-parties prenantes. Pis, des militants de la société civile ont été harcelés ou intimidés en raison de leur travail en faveur de la transparence. Ces militants bénéficient du soutien d'ONG du Nord, voire d'agences de coopération au développement. Certaines d'entre elles se demandent si elles n'ont pas joué avec le feu en mobilisant des organisations de la société civile dont les représentants ont ensuite été menacés dans leur intégrité physique par les autorités de leur pays. L'objectif à long terme est certes d'instaurer un dialogue démocratique dans des Etats autocratiques et de faire émerger des contre-pouvoirs, mais à quel prix pour les militants qui sont durement réprimés ?

Le rapport Un regard sur l'EITI relève un autre problème: les paiements individuels effectués par chaque entreprise apparaissent sous forme consolidée. Aussi est-il impossible d'identifier l'origine de «trous comptables». Pour PCQVP, il est essentiel de publier les chiffres pour chaque paiement et chaque encaissement, classés par entreprise et par type de revenus, comme l'a fait le Nigeria. Il serait en outre important d'exiger la publication des contrats d'investissement conclus entre les sociétés étrangères et les gouvernements hôtes. Les organisations de la société civile auraient ainsi la possibilité de connaître les conditions et les formules utilisées pour calculer les coûts et la distribution des bénéfices entre les firmes et le gouvernement.

Si l'EITI représente un pas dans la bonne direction, elle souffre de faiblesses majeures. Comme pour tout processus à caractère strictement volontaire, les signataires qui ne remplissent pas leurs engagements ne sont pas sanctionnés juridiquement. Il faudrait que les principes de l'EITI soient traduits dans la législation nationale pour leur conférer plus de force. En outre, la confidentialité qui entoure les contrats et le manque d'information désagrégée quant aux paiements des diverses industries extractives n'offrent qu'une transparence limitée aux acteurs de la société civile.

\section{Allocation budgétaire et financement du développement: I'exemple tchadien}

La transparence n'est pas un objectif en soi, mais un moyen qui vise in fine à lutter contre la malédiction des ressources par le biais d'une meilleure gestion des dépenses publiques. Le cas du Tchad fournit des enseignements intéressants. La Banque mondiale a accordé un prêt pour la construction de l'oléoduc Tchad-Cameroun et les infrastructures pétrolières de Doba, soit le plus grand investissement privé réalisé en Afrique subsaharienne. Le montant du crédit n'était pas très important d'un point de vue financier (293 millions de dollars

27 Publish What You Pay et Revenue Watch Institute, Un regard sur l'EITI. Perspectives de la société civile et recommandations concernant l'EITI, New York, Revenue Watch Institute, 2006, 32 p. 
sur un coût total de 4200 millions $)^{28}$ mais devait exercer une influence capitale sur l'utilisation de la manne pétrolière: le prêt est assorti d'un engagement du gouvernement tchadien quant à l'allocation des revenus du pétrole.

Revenons en 2003, lorsque le Parlement tchadien adopte la Loi 001 sous la pression des bailleurs de fonds. Ce texte prévoit alors que $72 \%$ des revenus soient alloués à des secteurs prioritaires pour le développement (santé, éducation, infrastructure), $10 \%$ soient bloqués sur un compte pour les générations futures et $4,5 \%$ aillent aux régions productrices. Des irrégularités apparaissent dès les premiers versements des compagnies pétrolières. Le gouvernement tchadien utilise en effet une partie des revenus pour l'achat d'armes. Or, la Banque mondiale tient à faire du «modèle tchadien» un succès, ce d'autant que la Revue des industries extractives mentionnée ci-dessus met l'institution au défi de prouver que ses engagements dans le secteur extractif contribuent au développement des pays bénéficiaires.

La crise couve, puis éclate en décembre 2005 lorsque le Parlement tchadien modifie la Loi 001. Le président Idriss Déby Itno fait ajouter les dépenses de sécurité et l'administration aux domaines prioritaires susceptibles d'être financés par les revenus pétroliers. Le Fonds pour les générations futures est dissous. Ce fonds, établi sur le modèle du Fonds pétrolier étatique norvégien, avait pour objectif non seulement de stériliser une partie des recettes d'exportation afin de réduire les risques de «syndrome hollandais», mais aussi de préparer la transition vers l'après-pétrole, lorsque le pic de production aura été atteint et que les réserves s'assécheront. Ce camouflet affecte directement la crédibilité de la Banque mondiale, qui stoppe ses programmes de crédits et s'engage dans des négociations avec le Tchad. Le 26 avril 2006, les deux parties se réconcilient autour d'un accord qui prévoit que $70 \%$ des revenus pétroliers seront affectés à la lutte contre la pauvreté. Les dépenses liées à la sécurité doivent être financées par le budget général. Le Fonds pour les générations futures n'est pas rétabli.

Comment expliquer cette victoire pour le président Déby? Il faut sans doute chercher la réponse dans les tensions géopolitiques qui secouent la région. Miavril 2006, les rebelles tchadiens attaquent la capitale, N'Djamena, et menacent le régime en place. La crainte de voir le pouvoir tchadien passer aux mains d'un nouveau régime favorable à Khartoum et hostile aux intérêts occidentaux a sans doute pesé dans la décision de la Banque mondiale de reprendre son programme de financement à des conditions beaucoup moins rigoureuses qu'en 2003.

\section{Potentiel et limites des initiatives multi-parties prenantes}

Les initiatives dites multi-stakeholders ou multi-parties prenantes (IMP) répondent à un déficit de gouvernance à l'ère de la mondialisation eu égard à la montée en puissance des grands acteurs économiques et financiers et à l'affaiblissement des Etats. Elles ont pour ambition de corriger certains des aspects les plus néfastes de la mondialisation à travers des engagements volontaires et non contraignants (soft law) d'acteurs étatiques et privés. Les IMP cherchent sou-

28 D. Mascré, Course aux hydrocarbures, crise du Darfour, déstabilisation régionale. Le Tchad entre jeux pétroliers et jeux guerriers, Institut Thomas More Working Papers, $n^{\circ}$ 9/FR, Paris; Bruxelles, Institut Thomas More, 2007. 
vent à parer aux échecs du marché dans la production et la préservation de biens publics globaux ${ }^{29}$.

Les IMP se multiplient depuis la fin de la guerre froide et réunissent des acteurs aussi divers que les institutions financières internationales, les ONG, des personnalités du show-business, de la finance ou de la politique, des compagnies pétrolières, des gouvernements de pays producteurs et des agences de coopération au développement. Par leur biais, les organisations de développement peuvent établir des relations avec des acteurs influents qu'elles ignoraient de manière plus ou moins volontaire dans le passé. Cela leur permet d'avoir un impact sur des questions capitales en termes de politiques de développement, questions sur lesquelles elles n'avaient aucune prise auparavant.

Les IMP ne contestent pas l'idéologie libérale ni la logique marchande. Au contraire, elles utilisent souvent les mécanismes du marché pour influencer le comportement des parties prenantes, notamment les investisseurs et les consommateurs ${ }^{30}$. Par rapport aux processus intergouvernementaux traditionnels, les IMP présentent l'avantage de la flexibilité et de la rapidité. Elles se distinguent par leur pragmatisme et sont mues par le souci de trouver des solutions concrètes à des problèmes rencontrés sur le terrain. Ces initiatives posent toutefois de sérieuses questions de gouvernance, de légitimité et d'efficacité. Leur faiblesse découle notamment de leur nature volontaire et non contraignante, et donc de l'absence de sanction juridique. Les IMP comptent sur les incitations du marché pour sanctionner les abus, en mobilisant les investisseurs et les consommateurs sur une problématique donnée. Dans le cas du pétrole, le consommateur est toutefois incapable d'exercer des pressions en boycottant un pays qui ne serait pas membre de l'EITI. Il ne peut que très difficilement obtenir des informations sur l'origine des produits pétroliers qu'il achète. Pour qu'il puisse exercer une pression sur les producteurs, comme c'est le cas pour le marché des diamants, il faudrait garantir la traçabilité du pétrole du lieu d'extraction à la vente au détail, ce qui nécessiterait le concours actif des maisons de négoce et des distributeurs. Les investisseurs peuvent par contre influencer le marché pétrolier à travers les conditions liées au financement de projets d'extraction ainsi que leur politique d'investissement «éthique».

John Ruggie, le représentant spécial du secrétaire général des Nations unies chargé de la question des droits de l'homme et des sociétés transnationales, souligne dans son rapport final que les IMP ont pour défi de prouver leur crédibilité et leur efficacité31. Or, comment mesurer l'efficacité des IMP? Comment en évaluer l'impact sur le terrain ainsi que l'effet d'entraînement sur d'autres initiatives? Oran Young propose d'articuler les critères d'évaluation des IMP autour de cinq questions ${ }^{32}$ : 1 . L'IMP contribue-t-elle à résoudre le problème qui a

29 Les premières IMP se sont mobilisées sur des problématiques environnementales (par exemple Commission mondiale sur les barrages, Fonds pour l'environnement mondial, Forest Stewartship Council, etc.).

30 Voir par exemple: W. Reinicke, «The Other World Wide Web: Global Public Policy Networks», Foreign Policy, Winter 1999-2000, pp. 44-57.

31 Assemblée générale des Nations unies, Les entreprises et les droits de l'homme. Analyse des normes internationales relatives à la responsabilité sociale et à la transparence des entreprises, doc. A/HRC/4/35, 19 février 2007, pp. 19-20.

32 O. Young, International Governance: Protecting the Environment in a Stateless Society, Ithaca, Cornell University Press, 1994. 
motivé sa création? 2. Les objectifs initiaux sont-ils atteints? 3. Les résultats se traduisent-ils par des dispositions appropriées dans l'ordre juridique et politique des pays membres? 4. L'IMP suscite-t-elle des pratiques sociales impliquant un investissement de temps, d'énergie et de ressources de la part de ses membres? 5. Les résultats sont-ils efficients, durables et solides? L'efficacité des IMP peut ainsi se mesurer à l'aune des changements de comportement qu'elles induisent chez les principales parties prenantes grâce à une meilleure compréhension d'une problématique donnée. Toutefois, même si les IMP prônent un nouveau mode de gouvernance en dehors du seul cadre étatique, leur impact dépend largement des gouvernements et de leur volonté politique de traduire leurs engagements dans la pratique.

Il ne faut pas oublier que les IMP fédèrent autour de principes et d'objectifs communs des parties prenantes aux intérêts et aux motivations fondamentalement divergents. Il est par conséquent naturel que les conflits d'intérêts se traduisent par de fortes tensions, notamment lorsque certaines parties sont déçues par le faible impact et le manque d'efficacité d'une IMP sur le terrain malgré les efforts entrepris. Il est alors fréquent que le conflit se cristallise sur des questions de gouvernance qui n'ont pas été éclaircies lors du lancement de l'initiative. Il s'agit par exemple de clarifier les critères d'admission des parties prenantes et d'exclusion des membres qui ne tiennent pas leurs engagements. Cela pose des questions de gouvernance: Qui prend les décisions, et comment? Quel poids politique donner à chaque partie dans les instances de gouvernance? Comment sélectionner les représentants de la «société civile» et du secteur privé ? Qui finance une IMP, et avec quel droit de regard?

Ces questions de gouvernance se sont récemment posées avec acuité dans le secteur extractif (par exemple pour les Principes volontaires sur la sécurité et les droits de l'homme ${ }^{33}$ ). Dans le cas de l'EITI, il a été demandé à un groupe consultatif international réunissant des gouvernements, des ONG, des entreprises et un gestionnaire d'actifs d'élaborer des mécanismes de gouvernance $a d$ hoc, sous la houlette du président de Transparency International. Ce groupe a proposé en 2006 que l'organe de gouvernance de l'EITI se compose de cinq représentants de la société civile, cinq représentants de l'industrie extractive, huit représentants de gouvernements et un représentant d'investisseur. Trentesept candidats de la société civile du Nord comme du Sud se sont annoncés et cinq d'entre eux ont été élus au Conseil de l'EITI lors de la Conférence de l'EITI à Oslo, en octobre $2006^{34}$. Il a été décidé de transférer le secrétariat de l'EITI de Londres (DFID) à une entité juridique autonome sise à Oslo et appuyée par l'Agence norvégienne pour la coopération en faveur du développement. L'avenir nous dira si ce modèle produit les résultats escomptés.

33 Les décisions prises par les membres de l'initiative des Principes volontaires relatifs à la sécurité et aux droits de l'homme en ce qui concerne l'admission et l'exclusion de ses membres (industries extractives) illustrent ces tensions de manière fort pertinente. Voir: The Voluntary Principles on Security and Human Rights Plenary Adopts Formal Participation Criteria, <http://www.voluntaryprinciples.org/ news/200705_VP_Participation.pdf $>$.

34 Voir : <http://www.EITIoslo.no>. 
Les IMP explorent de nouveaux modes de gouvernance. Dans le secteur extractif, elles dénotent une volonté partagée de contrer la malédiction des ressources. Hormis l'Azerbaïdjan et le Nigeria, le manque d'empressement de la plupart des pays producteurs de pétrole membres de l'EITI pour établir au niveau national les mécanismes minimaux requis pour la mise en œuvre de l'initiative laisse penser que la volonté politique fait encore trop souvent défaut. De plus, les mesures d'intimidation prises par quelques gouvernements à l'encontre de représentants de la société civile qui militent pour une plus grande transparence des revenus pétroliers montrent que certains pays membres n'ont aucune volonté de mettre en œuvre leurs engagements au titre de l'EITI. Le renforcement en cours des modalités d'admission et d'exclusion devrait contribuer à consolider la crédibilité de l'initiative mais pourrait exclure des pays pauvres à faible gouvernance pour lesquels l'initiative est particulièrement pertinente.

A l'instar d'autres initiatives visant une meilleure gestion des matières premières, l'EITI a été lancée avec le soutien politique et la participation de pays européens principalement. Les Etats-Unis, la Chine et l'Inde n'y participent pas alors que leurs marchés représentent des débouchés majeurs et que leur industrie d'extraction investit massivement en Afrique, souvent dans des contextes de forte instabilité politique. Leur non-participation réduit considérablement l'efficacité de l'EITI du fait que les membres pourraient hésiter à exercer une forte pression sur les pays producteurs récalcitrants qui peuvent quant à eux jouer sur la concurrence entre sociétés européennes, américaines et asiatiques.

La transparence des revenus promue par l'EITI n'est qu'un premier pas dans la bonne direction. Pour que la population en bénéficie et que la manne pétrolière contribue aux Objectifs du Millénaire pour le développement, encore faut-il que les revenus soient alloués de manière judicieuse. Les récentes péripéties au Tchad ont déçu les espoirs placés dans le mécanisme d'affectation des recettes du pétrole mis en place avec le concours de la Banque mondiale. Il s'est avéré illusoire d'utiliser la conditionnalité de l'aide comme arme pour conjurer la malédiction de l'or noir dans un contexte de faible gouvernance et d'instabilité régionale. Cette évolution semble donner raison aux ONG qui s'opposaient d'entrée de jeu à toute implication de la Banque mondiale dans le financement de l'oléoduc et des infrastructures pétrolières. Pour ces organisations, les conditions politiques, institutionnelles et sociales ne permettaient pas que l'argent du pétrole serve à lutter efficacement contre la pauvreté au Tchad.

Vu l'évolution de l'offre et de la demande, la pression pour extraire du pétrole dans des régions instables à faible gouvernance ne faiblira pas. La Banque mondiale n'a pas le pouvoir d'empêcher l'exploitation de l'or noir en refusant de s'associer au financement de projets pétroliers. Dès lors, les initiatives telles que l'EITI sont utiles et pertinentes. Elles permettent de mobiliser des acteurs influents pour faire en sorte que la manne pétrolière contribue à la lutte contre la pauvreté plutôt qu'à la guerre civile. Mais ces initiatives volontaires ne sont pas suffisantes.

Les marchés financiers détiennent le pouvoir d'influencer les gouvernements producteurs et les entreprises extractives de manière décisive. Il faut saluer le fait que plus de 70 fonds de pension et institutions financières gérant des avoirs 
de l'ordre de 12300 milliards de dollars se soient associés à une déclaration de soutien à l'EITI en octobre $2006^{35}$. Toutefois, le monde de la finance peut et doit jouer un rôle beaucoup plus affirmé. Il y va non seulement du sort des populations frappées par la malédiction des ressources, mais aussi de son intérêt bien compris. La transparence peut en effet contribuer à améliorer le climat d'investissement, à réduire les risques de corruption et à renforcer la sécurité des approvisionnements, avec des retombées positives pour les pays producteurs et les consommateurs comme pour les marchés financiers. Aussi, les fonds de pension, les banques et les assurances pourraient inclure des critères de transparence pour tout crédit, placement ou couverture de risques dans le secteur extractif, à l'instar des institutions financières internationales. Les autorités des grandes places boursières devraient inclure des dispositions semblables dans les règlements de publication pour les industries minières et pétrolières cotées en bourse, comme le suggère PCQVP. Les banques commerciales auraient par ailleurs intérêt à exiger plus de transparence sur les revenus lorsqu'elles octroient des prêts gagés sur les recettes anticipées de l'exploitation pétrolière.

La Norvège et le Royaume-Uni ont exercé un véritable leadership tant au niveau politique que par le soutien financier et administratif fourni par leurs agences de coopération au développement. La Suisse s'est quant à elle peu engagée dans ce domaine, sans doute faute d'expérience dans la gestion de matières premières dont elle est largement dépourvue. Le pays représente par contre une importante place financière et de négoce international. Aussi la Suisse pourrait-elle prendre l'initiative de mobiliser des acteurs clés de la finance et du commerce afin que ceux-ci puissent aider les pays pauvres qui ont la chance - et la malchance - de posséder des ressources abondantes à en extraire les moyens d'améliorer le bien-être de leur population. Les enjeux pour les pays producteurs comme pour les marchés financiers justifient un investissement substantiel en faveur d'une plus grande transparence et d'une meilleure allocation des revenus. Ces initiatives peuvent aider à conjurer la malédiction des ressources en accompagnant les profondes transformations sociales que toute «ruée vers l'or, jaune ou noir», ne manque jamais de susciter.

35 Les institutions financières suisses associées sont Ethos, Sarasin et Swiss Re Asset Management. 